\title{
Notfälle im HNO-Bereich
}

$\mathrm{M}$ edizinische Notfälle verlangen das ganze Können des Arztes und seiner Helfer. Dabei ist der Ausgang oft genug ungewiss. Auch Notfälle im HNO-ärztlichen Fachgebiet sind relativ häufig. Viele von ihnen stellen eine akute Bedrohung des Lebens dar. Daher sollte jeder Arzt - und insbesondere natürlich der Facharzt für HNO-Heilkunde, aber auch der Allgemeinmediziner und der Kinderarzt - mit dieser Problematik vertraut sein. Allen, die gelegentlich mit Notfallsituationen auf dem Gebiet der HNO-Erkrankungen konfrontiert werden, sollen folgende Artikel eine rasche Orientierung geben und vermittelnder Helfer sein.

Die Abgrenzung, was als Notfall zu gelten hat, kann verschieden getroffen werden. Hier wurde versucht, die wichtigsten Krankheitszustände aufzunehmen, derentwegen ein Patient erfahrungsgemäß auch außerhalb der Sprechstundenzeit ärztliche Hilfe sucht. Es handelt sich dabei nicht immer um Notfälle in dem Sinne, dass nur durch sofortiges Handeln ein schwerer Schaden abgewendet werden kann, teilweise werden auch banale Erkrankungen und Baga- tellfälle dargestellt. Die Symptomatik einer so genannten „Bagatellerkrankung“ ist der einer ernsten Erkrankung oft sehr ähnlich. Der Patient kann diese oft nicht unterscheiden und ist daher zu Recht beunruhigt. Er tut gut daran, in solchen Fällen einen Arzt zu konsultieren. Die wichtigste Aufgabe des Arztes ist es dann, die Situation hinsichtlich ihrer akuten Gefahr und der möglichen Gefährdung richtig einzustellen. Dies setzt jedoch eine exakte Diagnose voraus. Die Versicherung, dass keine lebensbedrohliche Erkrankung vorliegt, ist eine ebenso verantwortungsvolle ärztliche Leistung wie das Abwenden einer akuten Gefahr durch eine spezifische Therapie.

Jeder Notfallpatient kommt mit einem beherrschenden Symptom wie Blutung, Atemnot, plötzlicher Hörverlust oder mit einer präzisen Vorgeschichte in ärztliche Behandlung. Die Gesundheitsstörung wird z.B. durch Verletzungen, Verätzungen oder Fremdkörper verursacht. Ziel der Artikel war es nicht, eine lehrbuchmäßige Vollständigkeit zu erzielen, sondern die Erkrankungen, die sich um ein Symptom gruppieren, darzustellen und kurz abzuhandeln.

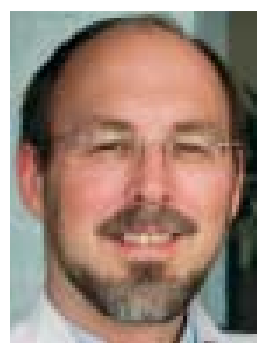

PD Dr. med. Joachim Müller, Würzburg

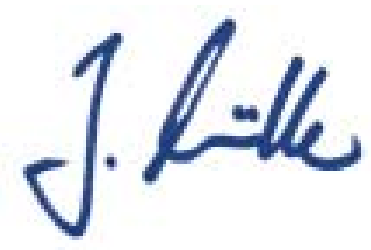

